\title{
New Mitral Annuloplasty Ring Enables Oversizing of Transcatheter Heart Valve and Prevents Central or Paravalvular Leakage
}

\author{
Georg Daniel Duerr ${ }^{1} \quad$ Jan-Malte Sinning ${ }^{2}$ Armin Georg Welz ${ }^{1} \quad$ Fritz Mellert $^{1}$ \\ ${ }^{1}$ Department of Cardiac Surgery, University Heart Center Bonn, \\ Bonn, Nordrhein-Westfalen, Germany \\ 2 Department of Medicine II-Cardiology, University Heart Center \\ Bonn, Bonn, Nordrhein-Westfalen, Germany \\ Address for correspondence Georg Daniel Duerr, Department of \\ Cardiac Surgery, University Heart Center Bonn, Sigmund-Freud-Str 25 \\ Bonn Nordrhein-Westfalen 5312, Germany \\ (e-mail: daniel.duerr@ukbonn.de; dduerr@uni-bonn.de).
}

Thorac Cardiovasc Surg 2017;65:460-466.

\begin{abstract}
Background Transcatheter valve-in-ring strategies have been developed to treat recurrent mitral regurgitation (MR) after failing surgical annuloplasty. However, suboptimal THV expansion with consecutive paravalvular leakage (PVL) is a procedure-immanent issue.

Methods A rigid, saddle-shaped ring was cut at four locations. The segments were reconnected with pull-springs, rearranged to the original shape, and covered with a sewing cuff. The length of the annuloplasty ring construct, including extended pullsprings, was defined by the perimeter of an appropriate THV. We deployed a Sapien XT within the new ring, expanded it to its maximum extent, and investigated the geometrical changes.

Results Fluoroscopy confirmed oval, saddle-shaped ring before dilation. After THV

Keywords

- mitral valve disease

- transcatheter aortic valve implantation

- valvular heart disease

- safety implantation, the ring segments spread apart and pull-springs were stretched. The extended ring changed its configuration from "oval" to "round" and anchored the THV leaving no paravalvular or central gaps as potential source for PVL.

Conclusion We developed an expandable annuloplasty ring that is perfectly concerted to THV implantation. This proof-of-concept study revealed no PVL and good oversizing ability that might impact future annuloplasty ring design. Further studies have to evaluate durability and device safety.
\end{abstract}

\section{Introduction}

In elderly patients suffering from ischemic cardiomyopathy, mitral regurgitation (MR) is a common valvular heart disease with increasing prevalence. ${ }^{1}$ Remodeling of the ischemic myocardium leads to dilation of the left ventricle and of the mitral annulus with consecutive papillary muscle displacement and therefore dysfunction. The tension on the mitral leaflets may result in restricted valvular closure and consecutive central regurgitation. ${ }^{2}$ Therapeutic options are surgical valve replacement or reconstruction techniques, depending on the underlying disease and reason for MR. $^{3}$ Surgical

received

August 21, 2016

accepted after revision

October 10, 2016

published online

February 10, 2017 reconstruction of the valve-so-called surgical ring annuloplasty-includes implantation of a mitral annuloplasty ring for the reduction in the mitral annular area and avoiding further dilation of the annulus. When added to conventional coronary artery bypass grafting surgery, mitral valve repair with restrictive annuloplasty has been shown to be a favorable treatment option for patients with ischemic secondary MR. ${ }^{4}$ Unfortunately, the success of surgical mitral annuloplasty is often limited by persistent or recurrent MR, especially in patients with ischemic, functional MR. A recent study revealed $58.8 \%$ recurrence of moderate or severe regurgitation within 2 years after surgical annuloplasty in ischemic MR (c) 2017 Georg Thieme Verlag KG Stuttgart - New York
DOI https://doi.org/ 10.1055/s-0037-1598029. ISSN 0171-6425. 
patients. ${ }^{5}$ In case of failure of surgical mitral annuloplasty, prosthetic valve replacement is necessary. However, this complex redo operation in a demanding patient population is often associated with significant morbidity and mortality.

In high-risk patients with aortic or pulmonary valve diseases, minimally invasive transcatheter strategies have been proven to be beneficial for the postoperative outcome and long-term survival. ${ }^{6-9}$ Further, it has been shown that transcatheter aortic valve-in-valve (VIV) implantation may obviate the need for redo surgery. ${ }^{10}$ Therefore, to improve survival and postoperative health of high-risk patients suffering from recurrent MR when surgical mitral annuloplasty has failed, minimally invasive transcatheter therapy is a desirable strategy. The actual data from the VIVID (Valve-in-Valve International Data) registry outlines that transcatheter mitral VIV (TMVIV) and transcatheter mitral valvein-ring (TMVIR) implantations are a less invasive approach and possibly an alternative for calling redo operations. On the other hand, different studies report difficulties in TMVIR procedure being based on different shape and material properties of mitral annuloplasty rings and transcatheter heart valves (THVs), often resulting in paravalvular or central leakage of the THV leading to moderate/severe residual MR ( - Video 1).$^{11-14}$ A further shortcoming of this new technique is, that expansion of the THV within the annuloplasty ring is limited by the shorter anteriorposterior diameter of the predominantly oval rings, preventing optimal THV oversizing. ${ }^{14,15}$ Oversizing of the THV is further associated with the risk of left ventricular outflow tract (LVOT) obstruction after VIR procedures. ${ }^{16}$ Multiple studies addressed these problems and described closure of residual leakage with plugs and occluders, which meant downsizing of the implanted THV in all reported cases. Also, different sizing strategies have been described. ${ }^{11,15,17,18}$ However, so far no study exists that proposes satisfactory solutions to these problems, and the overall results of TMVIR, summarized in the VIVID registry, report more than moderate regurgitation in $14.8 \%$ after TMVIR, but only $2.6 \%$ in TMVIV. Also, the composite endpoint of 30-day survival and freedom of more significant MR or clinically evident LVOT obstruction is only $71.6 \%$ for TMVIR, whereas it is $88.8 \%$ after TMVIV.

\section{Video 1}

Expansion of a Sapien XT in a naïve, oval, and rigid annuloplasty ring. THV implantation in a naïve, oval, and rigid annuloplasty ring reveals paravalvular gaps even after overexpansion of the THV. Online content including video sequences viewable at: https://www. thieme-connect.com/products/ejournals/html/doi/101055-s-0037-1598029-tcs-16-4598-v1.mp4.

In a recent publication, we presented a new surgical ring concept, which addresses these issues. ${ }^{19}$ We developed an expandable mitral annuloplasty ring that fully adjusts to THV expansion. The new annuloplasty ring is expanded in its diameter by THV dilation, and it changes its shape from oval to round. These ring properties provide better THV oversizing capacities and reduce the risk of paravalvular or central leakage.

In the present study, we demonstrate a modified, even simpler mitral annuloplasty device that provides similar expansion and reshaping properties as the reported annuloplasty ring. The current ring is constructed to facilitate TMVIR procedure and overcome the discussed pitfalls and problem, thus rendering TMVIR safer in high-risk patients with recurrent MR.

\section{Material and Methods}

\section{Ring Design}

A rigid and saddle-shaped annuloplasty ring (Profile 3D, Medtronic Inc., Minneapolis, United States) was cut at four predefined locations, for example at 3, 6, 9, and 12 o'clock. The four segments were then reconnected with small pullsprings, rearranged to the original shape conserving its three-dimensional (3D) and oval structure, and covered with a new sewing cuff (-Figs. 1A-D, -2A, B). The length of the annuloplasty ring construct, including the four ring segments and the completely extended pull-springs, was defined by the perimeter of an appropriate THV.

\section{Implantation of Transcatheter Heart Valve into Our New Annuloplasty Ring}

This new annuloplasty ring was introduced and fixed in a mock setup model of a heart, consisting of Styrofoam ( - Fig. 2C). Then, we deployed a 23-mm THV (Sapien XT, Edwards Life Sciences, Unterschleißheim, Germany) of larger perimeter within the new annuloplasty ring (-Fig. 2D). The correct positioning of the THV within the annuloplasty ring was confirmed (-Fig. 3A) and the crimped Sapien XT was then expanded stepwise (-Fig. 3B, C). During the process of TMVIR implantation, the conformational changes of the new annuloplasty ring were recorded simultaneously via fluoroscopy (-Video 2; Veradius mobile C-arm, Philips, Amsterdam, The Netherlands). To securely anchor the THV to the ring, the dilation balloon of the deployment catheter was expanded to its maximum extent (- Fig. 3D).

\section{Video 2}

Sapien XT implantation in our expandable annuloplasty ring. High-pressure balloon dilation of a 23-mm Sapien XT within our new annuloplasty ring shows conformational change from oval to round. Note the enlarged gaps between the four separated ring segments with expanded pull-springs. Online content including video sequences viewable at: https://www. thieme-connect.com/products/ejournals/html/doi/101055-s-0037-1598029-tcs-16-4598-v2.mp4.

\section{Results}

The implantation process of TMVIR was continuously monitored via fluoroscopy, as mentioned earlier. Before deployment of the Sapien XT valve into our new annuloplasty ring, 
A

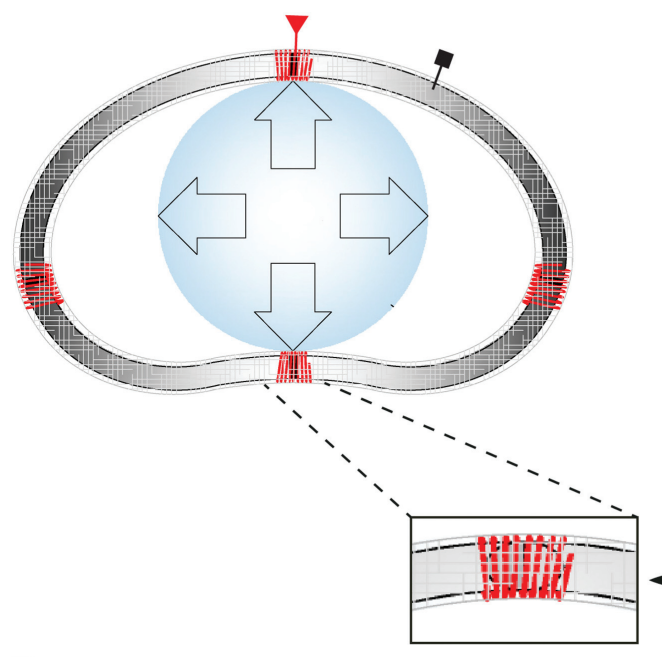

B

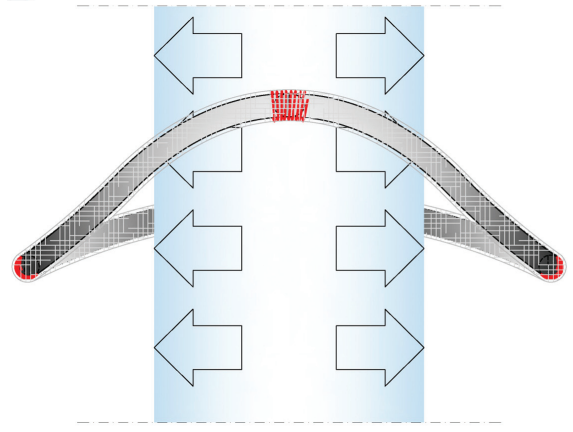

C

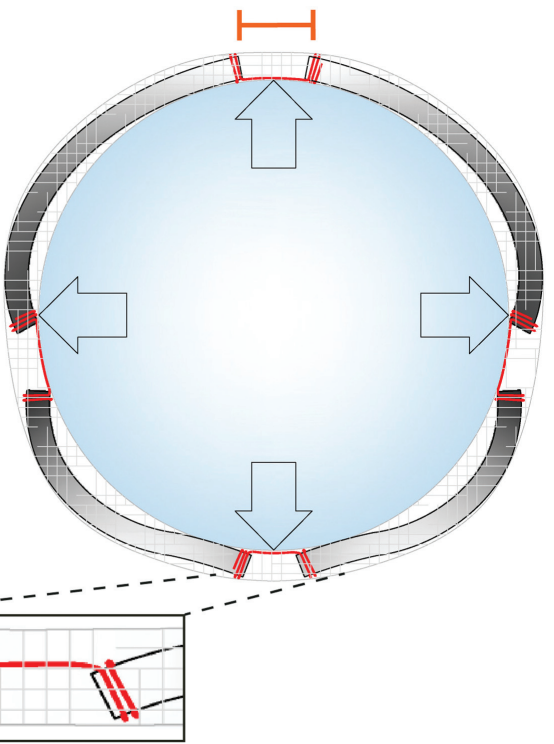

D

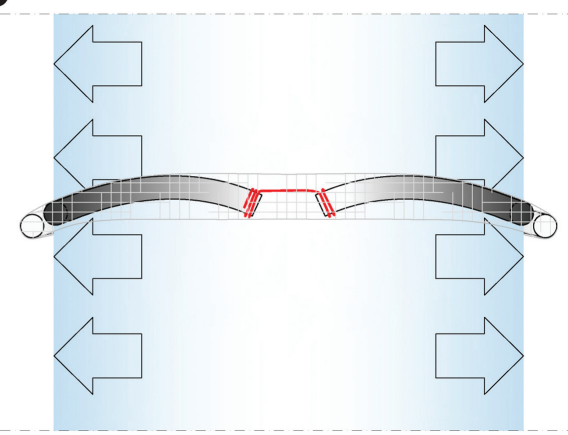

Fig. 1 True to scale construction plan of our new mitral annuloplasty ring. Panels A and B show transverse and coronal planes of the new annuloplasty ring before expansion of the dilation balloon. $C$ and D demonstrate the already expanded ring, leaving gaps (orange bracket in panel C) between the ring's segments (black square in panel A) and with stretched pull-springs connecting the segments. Red triangle in (A) indicates the upper predetermined breaking point; the red curved lines at 3, 6, 9, and 12 o'clock demonstrate the pull-springs.

the latter appeared oval and the correct position of the four connected ring segments in the ring's original, 3D conformation was clearly visible ( $\mathbf{F}$ Fig. $\mathbf{3 A}$ ). Before expansion of the $\mathrm{THV}$, the pull-springs appeared contracted, connecting the four ring segments tightly to each other.

During the implantation process of the THV, the four ring segments spread wide apart, leaving large gaps between them. Therefore, the ring expanded in a perfectly circular fashion. Oversizing by applying maximum pressure to the dilation balloon of the Ascendra delivery system (Edwards Lifesciences, Irvine, California, United States) caused the pull-springs to stretch. As a result, the new annuloplasty ring changed its configuration from "oval" to perfectly "round" shape. Thus, the ring's shorter diameter expanded from approximately 13 to 23 mm (-Fig. 3A-D; - Video 2). As a further result, the implanted $23 \mathrm{~mm}$ Sapien XT valve was securely attached to the new annuloplasty ring (-Fig. 4). No paravalvular or central gaps that could potentially cause MR were observed.

\section{Discussion}

It has been demonstrated that transcatheter aortic VIV implantation may obviate the need for redo surgery as did
TMVIV and TMVIR implantations. ${ }^{10}$ Taken into account the success of THV implantation in aortic and mitral position, THV implantation in VIR technique was suggested. ${ }^{9,20-22}$ Most studies showed improvement of hemodynamic parameters and postinterventional functional status, thus demonstrating feasibility of this TMVIR technique. ${ }^{1,23-26}$ Nevertheless, differences in geometry and structure of the utilized annuloplasty rings and THVs, respectively, raised unforeseen technical problems. ${ }^{13,14,19}$ This provoked intensive research on the optimal match between diverse commercially available annuloplasty rings and THVs. Different studies conducted in large animals and selected patients described multiple combinations of annuloplasty rings and THVs, respectively, and reported feasibility of VIR implantation in certain ring/THV combinations. ${ }^{12,26}$ However, some issues could not be addressed so far: The combination of some rings with THV devices does not allow appropriate oversizing of the THV within the annuloplasty ring. Furthermore, rigidity of the ring's material and its configuration, for example oval and saddle-shaped, resulted in more than mild remaining MR. This was caused by unexpected and undesirable morphologic changes of the expanded THV on the one hand, and paravalvular leakage (PVL) in the areas where the expanded, 

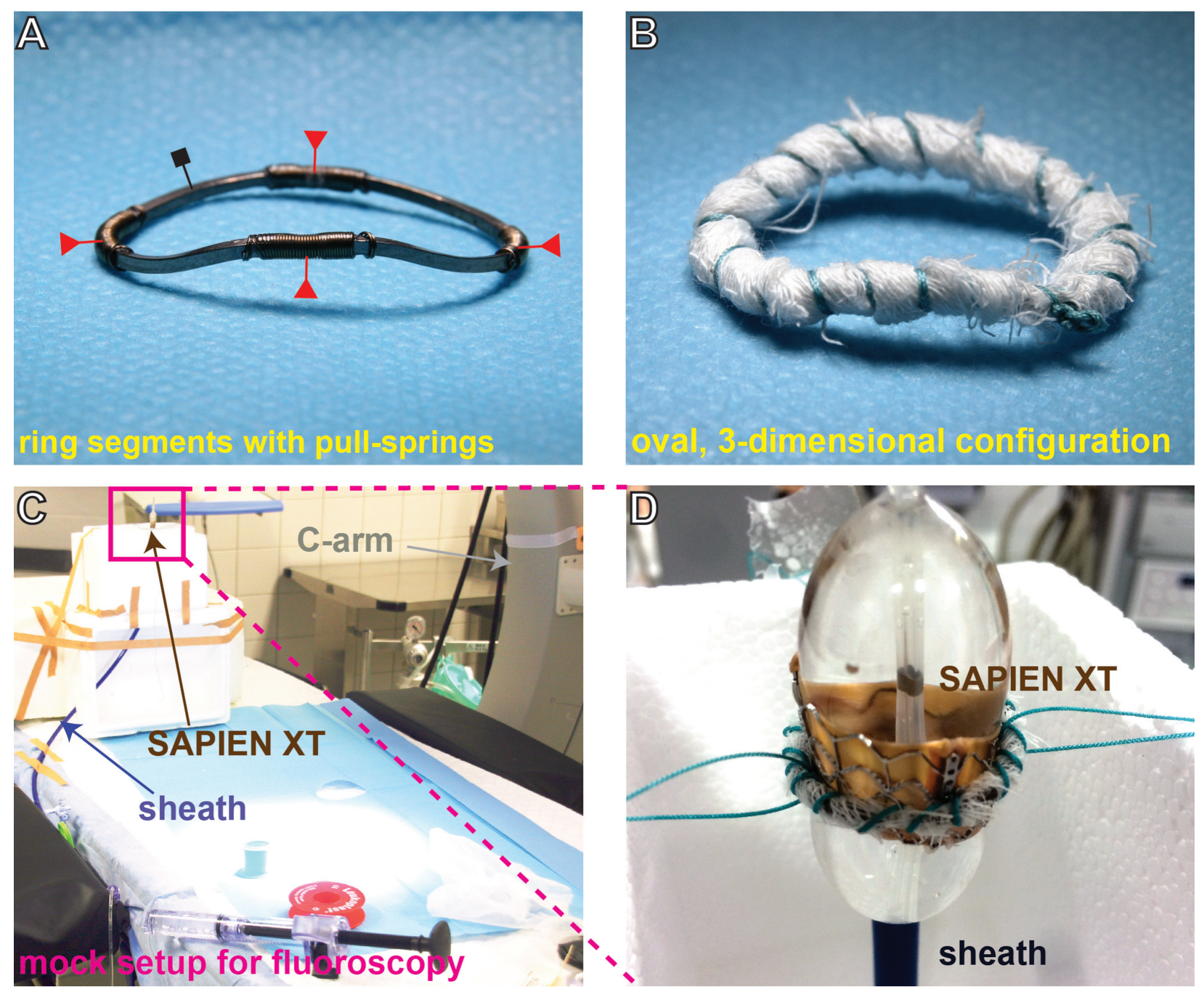

Fig. 2 Implantation of a Sapien XT THV in the new annuloplasty ring in a mock setup. Panel A shows a close-up picture of the reconstituted new annuloplasty ring with the four segments (black square) being connected with the four pull-sprigs (red triangles). (B) Oval, saddle-shaped new annuloplasty ring covered with the suture cuff. Panel C demonstrates the Styrofoam mock setup for VIR implantation. (D) Magnified detail of the Sapien XT after high-pressure balloon expansion within the new annuloplasty ring.

round THV does not seal the edges of the oval, longitudinal diameter of the THV on the other hand. ${ }^{13,14,19}$

Bapat addressed this problem of ring/THV mismatch and created an application (app) that indicates issues caused by configurational mismatch between different possible combinations of THVs and annuloplasty rings. According to this app, VIR procedure with the Sapien XT valve is not recommended for the frequently used rigid and oval, often also saddleshaped mitral annuloplasty rings (e.g., Edwards GeoForm ring, which has comparable geometrical and material properties as the Medtronic Profile 3D ring), due to the high risk of paravalvular leak and Sapien valve deformation. ${ }^{27}$

To overcome PVL, some interventionists used vascular occluders to seal paravalvular leaks between the circular THV and the longitudinal diameter edges of the oval annuloplasty ring in the same manner as after THV implantation in failing surgical bioprosthesis. ${ }^{28}$ Because of technical challenges of this strategy, it is not a favorable option in terms of a perfect configurational match between the THV stent frame and the original annulo- plasty ring. As the round configuration of a high-pressure balloon-expandable THV is essential for its durability and performance, the annuloplasty ring is the device whose shape and properties should be addressed. Various commercially available annuloplasty rings exist whose material properties range from rigid to flexible, and whose shape may be any combination of oval or D-shaped, and flat or saddle-shaped, 3D. In this variety of ring devices, THV implantation may be difficult due to inappropriate THV expansion-resulting in downsizing of the THV, as mentioned earlier. ${ }^{11,12,14,15,17}$

Therefore, we developed this new technical concept for an expandable and transformable mitral annuloplasty ring, which is tailored to provide perfect properties for subsequent THV implantation and oversizing. To fulfill these demands, we introduced four predefined breaking points into an oval, saddle-shaped Medtronic Profile 3D annuloplasty ring, permitting the ring to separate into four independent segments. The segments were then reconnected with pull-springs and the ring was rearranged to its original shape and covered with 

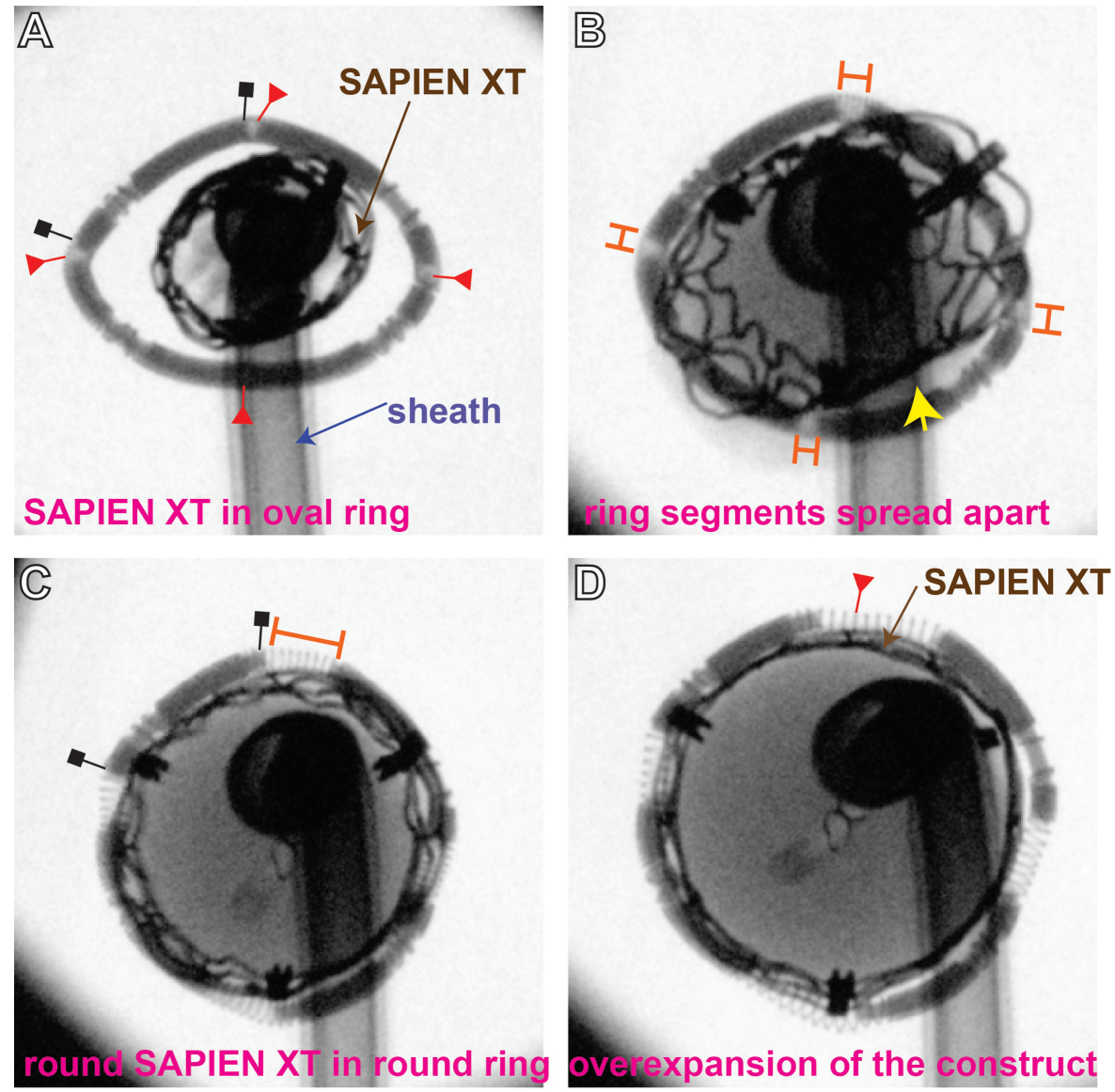

Fig. 3 Fluoroscopy-guided implantation of the Sapien XT within our new, expandable annuloplasty ring. Panel A shows a transverse view of the yet unexpanded, oval annuloplasty ring with adjacent ring segments (black squares define segment's dimension) and predefined braking points (red triangles). Note that the pull-springs are contracted. Panels B and C show the process of THV implantation in the new ring: Note the conformational change of the new ring from oval (B) to round (C) and the enlarging gaps between the segments (orange brackets in $B$ and $C$ ). Yellow arrowhead in $B$ indicates paravalvular gap between the yet incompletely expanded Sapien XT and the new ring. Panel D reveals the deployed Sapien XT in the expanded new annuloplasty ring after overexpansion. Note the enlarged gaps between the ring's segments and the stretched pull-springs (red triangle).

a new sewing cuff. The perimeter of the annuloplasty ring construct, including the extended pull-springs, was defined by the perimeter of an appropriately oversized THV. Subsequent VIR implantation with an Edwards SAPIEN XT $23 \mathrm{~mm}$ valve in the new annuloplasty ring was evaluated using fluoroscopy. The new properties of our expandable mitral annuloplasty ring prevented paravalvular and central leakage and permitted adequate oversizing of the THV within the new ring. Therefore, similar results may be expected with larger balloon-expandable valves.

In TMVIR procedures besides leakage, a major issue is the short landing zone so that depending on positioning of the valve, LVOT obstruction can occur. However, the radio-opaque landing zone of an annuloplasty ring might be a considerable advantage for the later deployment of a THV. Also, expansion and oversizing of a THV in the mitral annulus with possible lateral displacement of the native mitral valve anterior leaflet are associated with the risk of LVOT obstruction. ${ }^{16} \mathrm{~A}$ helpful tool to preventatively encounter these problems is the recently suggested "concept of the D-shaped annulus" assessment for transcatheter mitral valve implantation using multidetector computed tomography scans. It emphasizes the importance of measuring the LVOT dimension, mitral valve

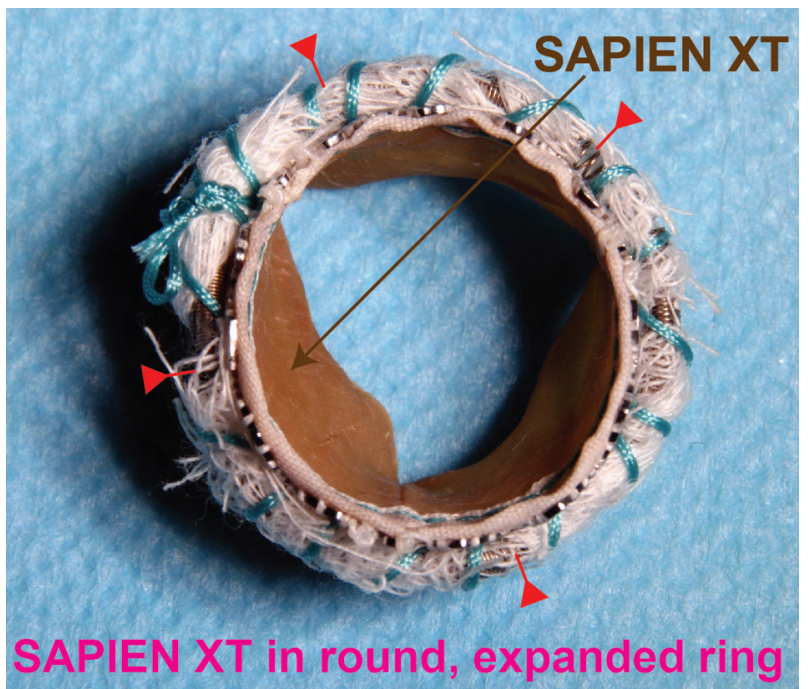

Fig. 4 Sapien XT and new annuloplasty ring after overexpansion. The expanded and round new annuloplasty ring serves as anchor for the Sapien XT $23 \mathrm{~mm}$ THV. The expanded THV/ring construct reveals perfect geometrical properties of the new ring in respect to THV implantation. Note the absence of paravalvular gaps between the Sapien XT and the new, expanded ring. Red triangles: stretched pullsprings connecting the four ring's segments. 
to LVOT angulation, septal thickness, and left ventricular to aortic angulation. ${ }^{29}$ In this respect, also new repositionable and retrievable devices for VIR implantation (e.g., Boston Lotus Valve System [Boston Scientific, Marlborough, Massachusetts, United States] or the Direct Flow valve [Direct Flow Medical, Santa Rosa, California, United States]) have to be evaluated and compared with results observed from balloonexpandable THV.

Further studies have to be conducted regarding safety and efficacy of our new annuloplasty ring. To assess the durability of our annuloplasty ring, material studies and fatigue testing (pulse duplicator) will be necessary to identify the best material for the pull-springs and their connection to the ring segments as well as the core segments of the ring. Furthermore, long-term studies in larger animals (e.g., pigs) have to evaluate device safety as well as the risk of LVOT obstruction and mitral annulus rupture in vivo.

Our new concept of an expandable mitral annuloplasty ring may be applied to the plethora of already existing and commercially available ring devices, and we suppose that this concept may also be transferable to different self-expanding devices after their perimeter-controlled pre-dilation. Taken into account that small surgical mitral valves are associated with a substantial risk of high gradients after THVIV implantation-as reported by the VIVID registry-our new concept could also be introduced into these surgical valves. This might qualify these smaller surgical mitral valves for subsequent implantation of larger THV devices. One limitation of our work is that we examined our new ring concept only in this single ex vivo study. Hence, our ring device has to be evaluated in vivo in large animals (pigs).

\section{Conclusion}

Surgical mitral annuloplasty with various ring devices is a valuable treatment option for patients with secondary and primary mitral valve regurgitations. Especially in patients with recurrent MR after surgical treatment of ischemic regurgitation, redo surgery with valve replacement may be associated with high morbidity and mortality. In this respect, minimally invasive transcatheter strategies have been tested. However, frequent issues encountered by TMVIR procedure are suboptimal THV expansion and considerable paravalvular or central leakage due to the oval configuration of the often rigid annuloplasty rings. This results in a demand for new generations of annuloplasty rings in the emerging field of VIR treatment. Hence, for better treatment of high-risk patients with recurrent MR, we developed an expandable annuloplasty ring that is perfectly concerted to THV implantation. Our proof of concept in a mock model revealed no paravalvular and central gaps, but great oversizing capacities. It could therefore inspire future annuloplasty ring design. Further in vivo studies in large animals have to evaluate device durability and safety in terms of LVOT obstruction or annulus rupture. Furthermore, this concept could be introduced in surgical valve bioprosthesis to enable later THV implantation without the need of downsizing.

\section{Note}

The data have been presented at the 45th annual meeting of The German Society for Thoracic and Cardiovascular Surgery in February 2016 as an oral presentation with the title: "New, Expandable Mitral Annuloplasty Ring Designed to Prevent Central or Paravalvular Leak and Provide Better THV-Oversizing Capacities," (Abstract-No.: A-760-0029-00499).

\section{Conflict of Interest}

All authors claim that there is no conflict of interest regarding this article.

\section{Acknowledgment}

We thank Dagmar Pasch for the expert assistance with the illustration of - Fig. 1.

\section{References}

1 Nkomo VT, Gardin JM, Skelton TN, Gottdiener JS, Scott CG, Enriquez-Sarano M. Burden of valvular heart diseases: a populationbased study. Lancet 2006;368(9540):1005-1011

2 Otsuji Y, Handschumacher MD, Liel-Cohen N, et al. Mechanism of ischemic mitral regurgitation with segmental left ventricular dysfunction: three-dimensional echocardiographic studies in models of acute and chronic progressive regurgitation. J Am Coll Cardiol 2001;37(2):641-648

3 Nishimura RA, Otto CM, Bonow RO, et al; American College of Cardiology/American Heart Association Task Force on Practice Guidelines. 2014 AHA/ACC guideline for the management of patients with valvular heart disease: a report of the American College of Cardiology/American Heart Association Task Force on Practice Guidelines. J Am Coll Cardiol 2014;63(22):e57-e185

4 Deja MA, Grayburn PA, Sun B, et al. Influence of mitral regurgitation repair on survival in the surgical treatment for ischemic heart failure trial. Circulation 2012;125(21):2639-2648

5 Goldstein D, Moskowitz AJ, Gelijns AC, et al; CTSN. Two-year outcomes of surgical treatment of severe ischemic mitral regurgitation. N Engl J Med 2016;374(4):344-353

6 Cribier A, Eltchaninoff H, Bash A, et al. Percutaneous transcatheter implantation of an aortic valve prosthesis for calcific aortic stenosis: first human case description. Circulation 2002; 106(24):3006-3008

7 Grube E, Laborde JC, Gerckens U, et al. Percutaneous implantation of the CoreValve self-expanding valve prosthesis in high-risk patients with aortic valve disease: the Siegburg first-in-man study. Circulation 2006;114(15):1616-1624

8 Duerr GD, Breuer J, Schiller W. A new transventricular approach for pulmonary valve implantation in a patient with severe valve disease after tetralogy-of-Fallot repair. Heart 2013;99(19):1469-1470

9 Neves PC, Paulo NS, Gama V, Vouga L. Transapical aortic valve and mitral valve in ring prosthesis implantation-a new advance in transcatheter procedures. Interact Cardiovasc Thorac Surg 2014; 19(2):344-346

10 Dvir D, Webb JG, Bleiziffer S, et al; Valve-in-Valve International Data Registry Investigators. Transcatheter aortic valve implantation in failed bioprosthetic surgical valves. JAMA 2014;312(2): $162-170$

11 Shuto T, Kondo N, Dori Y, et al. Percutaneous transvenous Melody valve-in-ring procedure for mitral valve replacement. J Am Coll Cardiol 2011;58(24):2475-2480

12 Kondo N, Shuto T, McGarvey JR, et al. Melody valve-in-ring procedure for mitral valve replacement: feasibility in four annuloplasty types. Ann Thorac Surg 2012;93(3):783-788 
13 Allende R, Doyle D, Urena M, et al. Transcatheter mitral "valve-inring" implantation: a word of caution. Ann Thorac Surg 2015; 99(4):1439-1442

14 Tavlasoglu M, Durukan AB, Kurkluoglu M. Can valved mitral prosthesis be implanted within all kinds of the mitral annuloplasty rings? Catheter Cardiovasc Interv 2013;81(1):172

15 Zou Y, Ferrari E, von Segesser LK. Off-pump transapical mitral valvein-ring implantation. Eur J Cardiothorac Surg 2013;43(4):849-855

16 Ghosh-Dastidar M, Narayana A, Boix R, Bapat V. Mitral valve-invalve and valve-in-ring for failing surgical bioprosthetic valves and rings. J Cardiovasc Surg (Torino) 2016;57(3):372-380

17 Kempfert J, Blumenstein J, Chu MW, et al. Minimally invasive offpump valve-in-a-ring implantation: the atrial transcatheter approach for re-operative mitral valve replacement after failed repair. Eur J Cardiothorac Surg 2009;35(6):965-969, discussion 969

18 Kliger C, Al-Badri A, Wilson S, et al. Successful first-in-man percutaneous transapical-transseptal Melody mitral valve-inring implantation after complicated closure of a para-annular ring leak. EuroIntervention 2014;10(8):968-974

19 Duerr GD, Sinning JM, Mellert F. New expandable mitral annuloplasty ring facilitates transcatheter mitral valve implantation: proof of concept. EuroIntervention 2016;11(14):e1662-e1668

$20 \mathrm{Al}$ Amri I, Debonnaire P, Witkowski T, et al. Mitral valve geometry and hemodynamics after surgical mitral valve annuloplasty and implications for percutaneous treatment of patients with recurrent mitral regurgitation. Am J Cardiol 2013;112(5):714-719

21 Petronio A, Giannini C, De Carlo M, Guarracino F. Antegrade percutaneous valve implantation for mitral ring dysfunction, a challenging case. Catheter Cardiovasc Interv 2012;80(4):700-703
22 Petronio AS, Giannini C, De Carlo M, Guarracino F. Rebuttal: Transcatheter valve in ring procedures may be safety and feasibility procedures in carefully selected patients. Catheter Cardiovasc Interv 2013;81(1):173

23 Bouleti C, Fassa AA, Himbert D, et al. Transfemoral implantation of transcatheter heart valves after deterioration of mitral bioprosthesis or previous ring annuloplasty. JACC Cardiovasc Interv 2015; 8(1 Pt A):83-91

24 Dahle G, Fiane AE, Rein KA. Transapical 29-mm Edwards SAPIENXT aortic valve in a 34-mm mitral annuloplasty ring. Innovations (Phila) 2012;7(4):290-294

25 Descoutures F, Himbert D, Maisano F, et al. Transcatheter valve-inring implantation after failure of surgical mitral repair. Eur J Cardiothorac Surg 2013;44(1):e8-e15

26 Wilbring M, Alexiou K, Tugtekin SM, et al. Pushing the limitsfurther evolutions of transcatheter valve procedures in the mitral position, including valve-in-valve, valve-in-ring, and valve-innative-ring. J Thorac Cardiovasc Surg 2014;147(1):210-219

27 Bapat V. Valve-in-valve apps: why and how they were developed and how to use them. EuroIntervention 2014;10(Suppl U): U44-U51

28 Hammerstingl C, Nickenig G, Endlich M, Mellert F, Schiller W. Treatment of a severely degenerated mitral valve bioprosthesis with simultaneous transapical paravalvular leak closure and valve-in-valve implantation. Eur Heart J 2012;33(15):1976

29 Blanke P, Dvir D, Cheung A, et al. A simplified D-shaped model of the mitral annulus to facilitate CT-based sizing before transcatheter mitral valve implantation. J Cardiovasc Comput Tomogr 2014; $8(6): 459-467$ 